\title{
Some properties of a new subclass of analytic univalent functions defined by multiplier transformation
}

\author{
Saurabh Porwal and Surya Pratap Singh
}

\begin{abstract}
The purpose of the present paper is to study the integral operator of the form

$$
\int_{0}^{z}\left\{\frac{I_{\mu}^{n} f(t)}{t}\right\}^{\delta} d t
$$

where $f$ belongs to the subclass $C(n, \alpha, \beta, \mu)$ and $\delta$ is a real number. We obtain integral characterization for the subclass $C(n, \alpha, \beta, \mu)$ and also prove distortion, rotation and radii theorem for this class. Relevant connections of the results presented here with various known results are briefly indicated.
\end{abstract}

Mathematics Subject Classification (2010): 30C45, 30C50, 30C55.

Keywords: Analytic, Univalent functions, Multiplier Transformation.

\section{Introduction}

Let $A$ denote the class of functions $f$ of the form

$$
f(z)=z+\sum_{k=2}^{\infty} a_{k} z^{k},
$$

which are analytic in the open unit disk $U=\{z: z \in \mathbb{C}$ and $|z|<1\}$ and satisfy the normalization condition $f(0)=f^{\prime}(0)-1=0$. Let $S$ be the subclass of $A$ consisting of functions of the form (1.1) which are also univalent in $U$.

A function $f$ of $S$ is said to be starlike of order $\alpha(0 \leq \alpha<1)$, denoted by $f \in S^{*}(\alpha)$, if and only if

$$
\Re\left\{\frac{z f^{\prime}(z)}{f(z)}\right\}>\alpha, z \in U,
$$


and is said to be convex of order $\alpha(0 \leq \alpha<1)$, denoted by $f \in K(\alpha)$, if and only if

$$
\Re\left\{1+\frac{z f^{\prime \prime}(z)}{f^{\prime}(z)}\right\}>\alpha, z \in U .
$$

The classes $S^{*}$ and $K$ of starlike and convex functions, respectively, are identified by $S^{*}(0) \equiv S^{*}$ and $K(0) \equiv K$.

These classes were first studied by Robertson [17].

In 2003 Cho and Srivastava [2], (see also [1]) introduced the multiplier transformation for functions $f$ of the form (1.1) as follows

$$
I_{\mu}^{n} f(z)=z+\sum_{k=2}^{\infty}\left(\frac{k+\mu}{1+\mu}\right)^{n} a_{k} z^{k} .
$$

For $\mu=1$, the operator $I_{\mu}^{n} \equiv I^{n}$ was studied by Uralegaddi and Somanatha [22] and for $\mu=0$ the operator $I_{\mu}^{n}$ reduce to well-known Sălăgean operator introduced by Sălăgean [19].

Using the multiplier transformation we introduce the class $S(n, \alpha, \mu)$ of functions of the form (1.1) satisfying the following condition

$$
\Re\left\{\frac{z\left(I_{\mu}^{n} f(z)\right)^{\prime}}{I_{\mu}^{n} f(z)}\right\}>\alpha, z \in U .
$$

It is worthy to note that for $\mu=0$ the class $S(n, \alpha, \mu)$ reduce to the class $S(n, \alpha)$ was first introduced by Sălăgean [19] and further studied by Kadioğlu [4].

It should be worthy to note that $S(0, \alpha, 0)=S^{*}(\alpha)$ and $S(1, \alpha, 0)=K(\alpha)$.

A function $f$ of $A$ belongs to the class $C(n, \alpha, \beta, \mu)$ if there exists a function $F \in S^{*}(\alpha)$ such that

$$
\left|\arg \frac{I_{\mu}^{n} f(z)}{F(z)}\right|<\frac{\beta \pi}{2}, z \in U,
$$

where $n \in N_{0}, 0 \leq \alpha<1,0<\beta \leq 1, \mu>-1$.

By specializing the parameters in $C(n, \alpha, \beta, \mu)$ we obtain the following known subclasses of $A$ studied earlier by various researchers.

(1) $C(0, \alpha, \beta, 0) \equiv C S^{*}(\alpha, \beta)$ studied by Mishra [9].

(2) $C(1, \alpha, \beta, 0) \equiv C(\alpha, \beta)$ studied by Mishra [9].

(3) $C(0,0, \beta, 0) \equiv C S^{*}(\beta)$ studied by Reade $[16]$.

(4) $C(1,0, \beta, 0) \equiv C(\beta)$ studied by Kaplan $[5]$.

(5) $C(0,0,1,0) \equiv S^{*}$ studied by Roberston [17], (see also [3], [21]).

(6) $C(1,0,1,0) \equiv K$ studied by Roberston [17], (see also [3], [21]).

In the present paper, we study the integral operator

$$
h(z)=\int_{0}^{z}\left\{\frac{I_{\mu}^{n} f(t)}{t}\right\}^{\delta} d t
$$

where $n \in N_{0}$ and $\delta$ is a real number. For $n=0$ and $n=1$ this integral operator was studied by Kim [6], Merkes and Wright [8], Mishra [9], Nunokawa([10], [11]), Pfaltzgraff [13], Royster [18], Patil and Tahakare [12] and Shukla and Kumar [20], (see also [15]). 
To prove our main results, we shall require the following definition and lemmas.

Definition 1.1. Let $P(\alpha)$ denote the class of functions of the form

$$
P(z)=1+\sum_{k=1}^{\infty} p_{k} z^{k}
$$

which are regular in $U$ and satisfy $\Re\{P(z)\}>\alpha, z \in U$.

Lemma 1.2. Let

$$
P(z)=1+\sum_{k=1}^{\infty} p_{k} z^{k}
$$

be analytic in $U$. If $\Re\{P(z)\}>\alpha$ in $U$, then

$$
\alpha\left(\theta_{2}-\theta_{1}\right)<\int_{\theta_{1}}^{\theta_{2}} \Re\left\{P\left(r e^{i \theta}\right)\right\} d \theta<2 \pi(1-\alpha)+\alpha\left(\theta_{2}-\theta_{1}\right),
$$

where $0 \leq \theta_{1}<\theta_{2} \leq 2 \pi, z=r e^{i \theta}$ and $0 \leq r<1$.

Proof. Since

$$
\Re\{P(z)\}>\alpha \text {. }
$$

It is easy to see that

$$
\left.(\Re\{P(z)\}-\alpha)\right|_{z=0}=1-\alpha .
$$

Then by mean value theorem, we have

$$
0 \leq \int_{\theta_{1}}^{\theta_{2}}\left(\Re\left\{P\left(r e^{i \theta}\right)\right\}-\alpha\right) d \theta \leq \int_{0}^{2 \pi}\left(\Re\left\{P\left(r e^{i \theta}\right)\right\}-\alpha\right) d \theta=2 \pi(1-\alpha) .
$$

or, equivalently

$$
0 \leq \int_{\theta_{1}}^{\theta_{2}}\left(\Re\left\{P\left(r e^{i \theta}\right)\right\}\right) d \theta-\alpha\left(\theta_{2}-\theta_{1}\right) \leq 2 \pi(1-\alpha),
$$

or

$$
\alpha\left(\theta_{2}-\theta_{1}\right)<\int_{\theta_{1}}^{\theta_{2}} \Re\left\{P\left(r e^{i \theta}\right)\right\} d \theta<2 \pi(1-\alpha)+\alpha\left(\theta_{2}-\theta_{1}\right) .
$$

The following lemma is a direct consequence of Lemma 1.2, and improves a result of Patil and Thakare ([12], Lemma 2.2).

Lemma 1.3. If $f \in S^{*}(\alpha)$, then

$$
\alpha\left(\theta_{2}-\theta_{1}\right)<\int_{\theta_{1}}^{\theta_{2}} \Re\left\{\frac{z f^{\prime}(z)}{f(z)}\right\} d \theta<2 \pi(1-\alpha)+\alpha\left(\theta_{2}-\theta_{1}\right),
$$

where $0 \leq \theta_{1}<\theta_{2} \leq 2 \pi, z=r e^{i \theta}$ and $0 \leq r<1$.

In the following lemma, we obtain integral characterization for the class $C(n, \alpha, \beta, \mu)$. 
Lemma 1.4. If $f \in C(n, \alpha, \beta, \mu)$, then

$$
-\beta \pi+\alpha\left(\theta_{2}-\theta_{1}\right)<\int_{\theta_{1}}^{\theta_{2}} \Re\left\{\frac{z\left(I_{\mu}^{n} f(z)\right)^{\prime}}{I_{\mu}^{n} f(z)}\right\} d \theta<\beta \pi+2 \pi(1-\alpha)+\alpha\left(\theta_{2}-\theta_{1}\right),
$$

where $0 \leq \theta_{1}<\theta_{2} \leq 2 \pi, z=r e^{i \theta}$ and $0 \leq r<1$. Conversely, let $f$ be analytic and satisfying $I_{\mu}^{n} f(z) \neq 0$ in $U$, if

$$
\int_{\theta_{1}}^{\theta_{2}} \Re\left\{\frac{z\left(I_{\mu}^{n} f(z)\right)^{\prime}}{I_{\mu}^{n} f(z)}\right\} d \theta>-\beta \pi+\alpha\left(\theta_{2}-\theta_{1}\right)
$$

then $f \in C(n, \alpha, \beta, \mu)$.

Proof. $f \in C(n, \alpha, \beta, \mu)$ implies that there exists a function $F \in S^{*}(\alpha)$ such that

$$
\left|\arg \frac{I_{\mu}^{n} f(z)}{F(z)}\right|<\frac{\beta \pi}{2}, z \in U .
$$

Therefore

$$
-\frac{1}{2} \beta \pi<\arg I_{\mu}^{n} f(z)-\arg F(z)<\frac{1}{2} \beta \pi .
$$

Let $0 \leq \theta_{1}<\theta_{2} \leq 2 \pi$. Then with $z=r e^{i \theta_{2}}$, we have

$$
-\frac{1}{2} \beta \pi<\arg I_{\mu}^{n} f\left(r e^{i \theta_{2}}\right)-\arg F\left(r e^{i \theta_{2}}\right)<\frac{1}{2} \beta \pi .
$$

and with $z=r e^{i \theta_{1}}$, we have

$$
-\frac{1}{2} \beta \pi<-\arg I_{\mu}^{n} f\left(r e^{i \theta_{1}}\right)+\arg F\left(r e^{i \theta_{1}}\right)<\frac{1}{2} \beta \pi .
$$

Combining (1.7) and (1.8), we obtain

$$
\begin{aligned}
-\beta \pi+\arg F\left(r e^{i \theta_{2}}\right)-\arg F\left(r e^{i \theta_{1}}\right) & <\arg I_{\mu}^{n} f\left(r e^{i \theta_{2}}\right)-\arg I_{\mu}^{n} f\left(r e^{i \theta_{1}}\right) \\
& <\beta \pi+\arg F\left(r e^{i \theta_{2}}\right)-\arg F\left(r e^{i \theta_{1}}\right),
\end{aligned}
$$

or

$$
-\beta \pi+\int_{\theta_{1}}^{\theta_{2}} d \arg F\left(r e^{i \theta}\right)<\int_{\theta_{1}}^{\theta_{2}} d \arg I_{\mu}^{n} f\left(r e^{i \theta}\right)<\beta \pi+\int_{\theta_{1}}^{\theta_{2}} d \arg F\left(r e^{i \theta}\right),
$$

or

$$
\begin{aligned}
-\beta \pi+\int_{\theta_{1}}^{\theta_{2}} \Re\left\{\frac{z F^{\prime}(z)}{F(z)}\right\} d \theta & <\int_{\theta_{1}}^{\theta_{2}} \Re\left\{\frac{z\left(I_{\mu}^{n} f(z)\right)^{\prime}}{I_{\mu}^{n} f(z)}\right\} d \theta \\
& <\beta \pi+\int_{\theta_{1}}^{\theta_{2}} \Re\left\{\frac{z F^{\prime}(z)}{F(z)}\right\} d \theta .
\end{aligned}
$$

But $F \in S^{*}(\alpha)$, then using Lemma 1.3 in (1.9), we have

$$
-\beta \pi+\alpha\left(\theta_{2}-\theta_{1}\right)<\int_{\theta_{1}}^{\theta_{2}} \Re\left\{\frac{z\left(I_{\mu}^{n} f(z)\right)^{\prime}}{I_{\mu}^{n} f(z)}\right\} d \theta<\beta \pi+2 \pi(1-\alpha)+\alpha\left(\theta_{2}-\theta_{1}\right)
$$

and this completes the proof of direct part of the lemma. 
To prove the converse part, we follow the techniques of Kaplan [5] and Patil and Thakare [12] and can obtain the desired result.

Remark 1.5. If we put $n=1, \mu=0$ in Lemma 1.4, we obtain the following result If $f \in C(\alpha, \beta)$, then

$$
-\beta \pi+\alpha\left(\theta_{2}-\theta_{1}\right)<\int_{\theta_{1}}^{\theta_{2}} \Re\left\{1+\frac{z f^{\prime \prime}(z)}{f^{\prime}(z)}\right\} d \theta<\beta \pi+2 \pi(1-\alpha)+\alpha\left(\theta_{2}-\theta_{1}\right),
$$

where $0 \leq \theta_{1}<\theta_{2} \leq 2 \pi, z=r e^{i \theta}$ and $0 \leq r<1$. Conversely, let $f$ be analytic and satisfying $f^{\prime}(z) \neq 0$ in $U$, if

$$
\int_{\theta_{1}}^{\theta_{2}} \Re\left\{1+\frac{z f^{\prime \prime}(z)}{f^{\prime}(z)}\right\} d \theta>-\beta \pi+\alpha\left(\theta_{2}-\theta_{1}\right)
$$

then $f \in C(\alpha, \beta)$.

\section{Main results}

Theorem 2.1. If $f \in C(n, \alpha, \beta, \mu)$, then $h \in C(\eta, \gamma)$, provided

$$
\frac{-\gamma}{\beta+2(1-\alpha)} \leq \delta \leq \frac{\gamma+2(1-\eta)}{\beta+2(1-\alpha)} .
$$

The result is sharp when (i) $\gamma=0$ (ii) $\eta=0, \gamma=1$.

Proof. From relation (1.3) we have

$$
h^{\prime}(z)=\left\{\frac{I_{\mu}^{n} f(z)}{z}\right\}^{\delta}
$$

Applying logarithmic differentiation and then taking real parts of both sides, we obtain

$$
\operatorname{Re}\left\{1+\frac{z h^{\prime \prime}(z)}{h^{\prime}(z)}\right\}=\delta \operatorname{Re}\left\{\frac{z\left(I_{\mu}^{n} f(z)\right)^{\prime}}{I_{\mu}^{n} f(z)}\right\}+(1-\delta) .
$$

For $\delta>0$, using Lemma 1.4, we get

$$
\begin{aligned}
\int_{\theta_{1}}^{\theta_{2}} \operatorname{Re}\left\{1+\frac{z h^{\prime \prime}(z)}{h^{\prime}(z)}\right\} d \theta & =\delta \int_{\theta_{1}}^{\theta_{2}} \operatorname{Re}\left\{\frac{z\left(I_{\mu}^{n} f(z)\right)^{\prime}}{I_{\mu}^{n} f(z)}\right\} d \theta+(1-\delta)\left(\theta_{2}-\theta_{1}\right) \\
& >\delta\left[-\beta \pi+\alpha\left(\theta_{2}-\theta_{1}\right)\right]+(1-\delta)\left(\theta_{2}-\theta_{1}\right) \\
& =-\beta \delta \pi+[1-(1-\alpha) \delta]\left(\theta_{2}-\theta_{1}\right) .
\end{aligned}
$$

To prove that $h \in C(\eta, \gamma)$, we have to show that the right hand side of the above inequality is not less than $-\gamma \pi+\eta\left(\theta_{2}-\theta_{1}\right)$, provided

$$
0 \leq \delta \leq \frac{\gamma+2(1-\eta)}{\beta+2(1-\alpha)}
$$


Similarly, for $\delta<0$, using Lemma 1.4, we get

$$
\int_{\theta_{1}}^{\theta_{2}} \Re\left\{1+\frac{z h^{\prime \prime}(z)}{h^{\prime}(z)}\right\} d \theta>\delta\left[\beta \pi+2(1-\alpha)+\alpha\left(\theta_{2}-\theta_{1}\right)\right]+(1-\delta)\left(\theta_{2}-\theta_{1}\right) .
$$

To show that $h \in C(\eta, \gamma)$, we have to prove that the right-hand side of the above inequality is not less than $-\gamma \pi+\eta\left(\theta_{2}-\theta_{1}\right)$, provided

$$
\frac{-\gamma}{\beta+2(1-\alpha)} \leq \delta \leq 0 \text {. }
$$

Combining (2.2) and (2.3), we obtain (2.1).

Thus the proof of Theorem 2.1 is established.

To show the sharpness, let us take the function $f(z)$ defined by the relation

$$
I_{\mu}^{n} f(z)=\frac{z}{(1-z)^{2(1-\alpha)+\beta}},
$$

then it is easy to see that this function belongs to $C(n, \alpha, \beta, \mu)$ with respect to the function $\frac{z}{(1-z)^{2(1-\alpha)}}$ belonging to $S^{*}(\alpha)$. Then

$$
h(z)=\int_{0}^{z} \frac{d t}{(1-t)^{[2(1-\alpha)+\beta] \delta}}
$$

and from condition (1.11) this functions belongs to $C(0,1)$ if and only if

$$
\frac{-1}{2(1-\alpha)+\beta} \leq \delta \leq \frac{3}{2(1-\alpha)+\beta} .
$$

Again for $\gamma=0$, from (2.5) we have

$$
1+\frac{z h^{\prime \prime}(z)}{h^{\prime}(z)}=\frac{1+\left[1-2\left(1-\frac{\{2(1-\alpha)+\beta\} \delta}{2}\right)\right] z}{1-z}
$$

and $\Re\left\{1+\frac{z h^{\prime \prime}(z)}{h^{\prime}(z)}\right\}>\eta$ if and only if

$$
1-\frac{\{2(1-\alpha)+\beta\} \delta}{2} \geq \eta \Rightarrow 0 \leq \delta \leq \frac{2(1-\eta)}{\beta+2(1-\alpha)} .
$$

Remark 2.2. The undermentioned results are particular cases of Theorem 2.1.

(i) If we put $n=0$ and $n=1$ with $\mu=0$ in Theorem 2.1 we obtain the corresponding results of Mishra [9].

(ii) If we put $n=1, \beta=0, \gamma=0$ with $\mu=0$ we obtain a result of Patil and Thakare $[12]$.

(iii) If we put $n=1, \beta=0, \eta=0$ with $\mu=0$ we obtain a result of Patil and Thakare $[12]$.

(iv) If we put $n=1, \alpha=0, \eta=0$ with $\mu=0$ we obtain a result of Patil and Thakare $[12]$.

(v) If we put $n=0, \beta=0, \eta=0$ we obtain a result of Patil and Thakare [12].

(vi) If we put $n=1, \alpha=0, \beta=0, \eta=0$ and $\gamma=1$ with $\mu=0$ we obtain a result of Nunokawa [11] as well as that of Merkes and Wright [8].

(vii) If we put $n=0, \alpha=0, \beta=0, \eta=0$ and $\gamma=1$ with $\mu=0$ we obtain a result of Nunokawa [11] as well as that of Merkes and Wright [8]. 
(viii) If we put $n=1, \alpha=0, \beta=1, \eta=0$ and $\gamma=1$ with $\mu=0$ we obtain a result of Nunokawa [11] as well as that of Merkes and Wright [8].

(ix) If we put $n=0, \alpha=0, \eta=0$ with $\mu=0$ we obtain a result of Shukla and Kumar $[20]$.

(x) If we put $n=0, \alpha=0, \beta=1, \eta=0$ and $\gamma=1$ with $\mu=0$ we obtain a result of $\operatorname{Kim}[6]$.

(xi) If we put $n=0, \alpha=1 / 2, \beta=0, \eta=0$ and $\gamma=1$ with $\mu=0$ we obtain a result of Nunokawa [11] as well as that of Merkes and Wright [8].

Theorem 2.3. Let $f \in C(n, \alpha, \beta, \mu)$. Then for $|z|=r$

$$
\frac{r(1-r)^{\beta}}{(1+r)^{\beta+2(1-\alpha)}} \leq\left|I_{\mu}^{n} f(z)\right| \leq \frac{r(1+r)^{\beta}}{(1-r)^{\beta+2(1-\alpha)}}
$$

The result is sharp.

Proof. By definition $f \in C(n, \alpha, \beta, \mu)$ if and only if there exists a function $P \in P(0)$ and $F(z) \in S^{*}(\alpha)$ such that

Therefore

$$
\frac{I_{\mu}^{n} f(z)}{F(z)}=[P(z)]^{\beta} .
$$

$$
\left|I_{\mu}^{n} f(z)\right|=|P(z)|^{\beta}|F(z)| \text {. }
$$

Now using the well-known inequalities (see [3])

$$
\frac{1-r}{1+r} \leq|P(z)| \leq \frac{1+r}{1-r}
$$

and

$$
\frac{r}{(1+r)^{2(1-\alpha)}} \leq|F(z)| \leq \frac{r}{(1-r)^{2(1-\alpha)}},
$$

we obtain the required inequalities.

Sharpness follows if we take $f(z)$ connected by the relation

$$
I_{\mu}^{n} f(z)=\frac{z(1+z)^{\beta}}{(1-z)^{\beta+2(1-\alpha)}}
$$

and

$$
F(z)=\frac{z}{(1-z)^{2(1-\alpha)}}
$$

Theorem 2.4. If $f \in C(n, \alpha, \beta, \mu)$, then

$$
\left|\arg \frac{I_{\mu}^{n} f(z)}{z}\right| \leq \beta \sin ^{-1} \frac{2 r}{1+r^{2}}+2(1-\alpha) \sin ^{-1} r .
$$

The result is sharp.

Proof. If $f \in C(n, \alpha, \beta, \mu)$, then

$$
\frac{I_{\mu}^{n} f(z)}{F(z)}=[P(z)]^{\beta},
$$

for some $P(z) \in P(0)$ and $F(z) \in S^{*}(\alpha)$. 
Thus

$$
\left|\arg \frac{I_{\mu}^{n} f(z)}{z}\right| \leq \alpha|\arg P(z)|+\left|\arg \frac{F(z)}{z}\right| .
$$

Now using the well-known results

$$
|\arg P(z)| \leq \sin ^{-1} \frac{2 r}{1+r^{2}}
$$

and a result of Pinchuk [14]

$$
\left|\arg \frac{F(z)}{z}\right| \leq 2(1-\alpha) \sin ^{-1} r,
$$

Using (2.7) and (2.8) in (2.6) we get the required result.

Sharpness follows if we take $f(z)$ to be the same as in Theorem 2.3.

Theorem 2.5. If $f \in C(n, \alpha, \beta, \mu)$, then $f \in S(n)$ for $|z|<r_{0}$, where

$$
r_{0}=\frac{(1+\beta-\alpha)-\sqrt{\alpha^{2}-2 \beta \alpha+\beta(2+\beta)}}{1-2 \alpha} \text {, when } \alpha \neq 1 / 2
$$

and

$$
r_{0}=\frac{1}{1+2 \beta}, \text { when } \alpha=1 / 2
$$

The result is sharp.

Proof. $f \in C(n, \alpha, \beta, \mu)$, if and only if there there exists a function $P \in P(0)$ and $F(z) \in S^{*}(\alpha)$ such that

$$
\begin{gathered}
\frac{I_{\mu}^{n} f(z)}{F(z)}=[P(z)]^{\beta} . \\
I_{\mu}^{n} f(z)=[P(z)]^{\beta} F(z) .
\end{gathered}
$$

Logarithmic differentiation of (2.9) yields

$$
\frac{z\left(I_{\mu}^{n} f(z)\right)^{\prime}}{I_{\mu}^{n} f(z)}=\beta \frac{z P^{\prime}(z)}{P(z)}+\frac{z F^{\prime}(z)}{F(z)} .
$$

Now by a result of MacGregor [7], we know that

$$
\left|\frac{z P^{\prime}(z)}{P(z)}\right| \leq \frac{2 r}{1-r^{2}}
$$

Therefore

$$
\begin{aligned}
\Re\left\{\frac{z\left(I_{\mu}^{n} f(z)\right)^{\prime}}{I_{\mu}^{n} f(z)}\right\} & \geq \Re\left\{\frac{z F^{\prime}(z)}{F(z)}\right\}-\beta\left|\frac{z P^{\prime}(z)}{P(z)}\right| \\
& \geq \frac{1-(1-2 \alpha) r}{1+r}-\beta\left(\frac{2 r}{1-r^{2}}\right) \\
& =\frac{(1-2 \alpha) r^{2}-2(1+\beta-\alpha) r+1}{1-r^{2}} .
\end{aligned}
$$

The right hand side of the above inequality is not less than or equal to zero provided $|z|=r<r_{0}$, where $r_{0}$ is as given in the statement of theorem. Sharpness follows if we take $f(z)$ to be the same as in Theorem 2.3 . 
Acknowledgement. The authors are thankful to the referee for his/her valuable comments and suggestions.

\section{References}

[1] Cho, N.E., Kim, T.H., Multiplier transformations and strongly close-to-convex functions, Bull. Korean Math. Soc., 40(2003), no. 3, 399-410.

[2] Cho, N.E., Srivastava, H.M., Argument estimates of certain analytic functions defined by a class of multiplier transformations, Math. Comput. Modell., 37(2003), no. 1-2, 39-49.

[3] Goodman, A.W., Univalent Functions, Vol. I and II, Mariner Publishing Company, Florida, 1983.

[4] Kadioğlu, E., On subclass of univalent functions with negative coefficients, Appl. Math. Comput., 146(2003), 351-358.

[5] Kaplan, W., Close-to-Convex Schlicht functions, Mich. Math. J., 1(1952), 169-185.

[6] Kim, Y.J., Univalence of Certain Integrals, Ph.D. Thesis, University of Cincinnati, 1972.

[7] MacGregor, T.H., Univalent power series whose coefficients have monotonic properties, Math. Z., 112(1969), 222-228.

[8] Merkes, E.P., Wright, D.J., On the univalence of certain integrals, Proc. Amer. Math. Soc., 27(1971), 97-100.

[9] Mishra, R.S., Geometrical and Analytical Properties of Certain Classes Related to Univalent Functions, Ph.D. Thesis, C.S.J.M. University, Kanpur, India, 2007.

[10] Nunokawa, M., On the univalence of certain integral, Proc. Japan Acad., 45(1969), 841845.

[11] Nunokawa, M., On the univalence of certain integral, Trans. Amer. Math. Soc., 146(1969), 439-440.

[12] Patil, D.A., Thakare, N.K., On univalence of certain integral, Indian J. Pure Appl. Math., 11 (1980), 1626-1642.

[13] Pfaltzgraff, J.A., Univalence of the integral of $f^{\prime}(z)^{\lambda}$, Bull. Lon. Math. Soc., 7(1975), 254-256.

[14] Pinchuk, B., On starlike and convex functions of order $\alpha$, Duke Math. J., 35(1968), 721-734.

[15] Porwal, S., Mapping properties of an integral operator, Acta Univ. Apul., 27(2011), 151155.

[16] Reade, M.O., On close-to-convex univalent functions, Mich. Math. J., 3(1955-56), 59-62.

[17] Robertson, M.S., On the theory of univalent functions, Ann. Math., 37(1936), 374-408.

[18] Royster, W.C., On the univalence of certain integral, Mich. Math. J., 12(1965), 385-387.

[19] Sălăgean, G.S., Subclasses of univalent functions, Complex Analysis-Fifth Romanian Finish Seminar, Bucharest, 1(1983), 362-372.

[20] Shukla, S.L., Kumar, V., Remarks on a paper of Patil and Thakare, Indian J. Pure Appl. Math., 13(1982), 1513-1525.

[21] Silverman, H., Univalent functions with negative coefficients, Proc. Amer. Math. Soc., 51(1975), 109-116.

[22] Uralegaddi, B.A., Somanatha, C., Certain Classes of Univalent Functions, in Current Topics in Analytic Function Theory, 371-374, World Sci. Publishing, River Edge, NJ. 
Saurabh Porwal

Department of Mathematics

UIET, CSJM University, Kanpur-208024

(U.P.), India

e-mail: saurabhjcb@rediffmail.com

Surya Pratap Singh

Department of Mathematics

UIET, CSJM University, Kanpur-208024

(U.P.), India 